Complex Analysis/Mathematical Analysis

\title{
Maximum modulus points and zero sets of entire functions of regular growth
}

\author{
Iossif Ostrovskii $^{\mathrm{a}, \mathrm{b}}$, Ersin Üreyen $^{\mathrm{a}}$ \\ ${ }^{a}$ Department of Mathematics, Bilkent University, 06800 Bilkent, Ankara, Turkey \\ ${ }^{\mathrm{b}}$ B. Verkin Institute for Low Temperature Physics and Engineering, 61103 Kharkov, Ukraine
}

Received 1 March 2005; accepted after revision 1 September 2005

Presented by Jean-Pierre Kahane

\begin{abstract}
We obtain lower asymptotic at $\infty$ estimates of the distance between a maximum modulus point and zero set of an entire function provided that the function is of regular growth with respect to a proximate order. The more regular the growth is the better the estimates are, and they are sharp in some sense. The case of infinite order is also considered; in this case a suitable analogue of usual proximate order is exploited. To cite this article: I. Ostrovskii, E. Üreyen, C. R. Acad. Sci. Paris, Ser. I 341 (2005). (C) 2005 Académie des sciences. Published by Elsevier SAS. All rights reserved.

\section{Résumé}

Points de module maximal et ensemble de zéros des fonctions entières de croissance regulière. Nous obtenons des estimations inférieure asymptotiques à l'infini de la distance entre un point de module maximal et l'ensemble des zéros d'une fonction entière, quand la fonction est supposée de croissance régulière par rapport à un ordre précisé. Les estimations s'améliorent avec la régularité, et dans un sens elles sont précises. Le cas d'ordre infini est aussi consideré ; dans ce cas nous avons utilisé une analogie appropriée de l'ordre précisé habituel. Pour citer cet article : I. Ostrovskii, E. Üreyen, C. R. Acad. Sci. Paris, Ser. I 341 (2005). (C) 2005 Académie des sciences. Published by Elsevier SAS. All rights reserved.
\end{abstract}

\section{Version française abrégée}

Un point de module maximal d'une fonction entière $f$ est un point $w \in \mathbf{C}$ tel que l'on ait (1). Les estimations inférieures asymptotiques (à l'infini) de la distance $R(w, f)$ entre $w$ et l'ensemble des zéros de $f$ jouent un rôle important dans la version de la théorie de Wiman-Valiron due à Macintyre [4] et aussi dans d'autres généralisations et applications. Les estimations de Macintyre sont valables en dehors d'un ensemble de valeurs exceptionelles de $|w|$ et sont exprimées en termes de $M(r, f)$. Dans [5] nous avons obtenu des estimations qui, à la différence de celles de Macintyre, sont valables pour tout $|w|$ suffisamment grand et exprimées en termes des ordres precisés.

Comme dans presque toutes les applications de la théorie des fonctions entières (voir, e.g., [3]) on considère des fonctions à la croissance régulière, il est naturel de chercher des estimations de $R(w, f)$ pour de telles fonctions $f$.

E-mail addresses: iossif@fen.bilkent.edu.tr (I. Ostrovskii), ureyen@ fen.bilkent.edu.tr (E. Üreyen). 
Dans [5], nous avons traité ce sujet seulement pour les fonctions d'ordre positif fini. Dans cette Note, nous obtenons des résultats plus généraux, applicables aussi aux fonctions d'ordre zéro ou infini et qui sont plus précis dans un sens.

Soient $0 \leqslant \rho<\infty$ et $\rho(r)$ un ordre précisé (o.p.) fort au sens de Levin ([3], pp. 39-41), c'est-à-dire, $\rho(r)$ est une fonction $\rho(r) \in C^{2}\left(\mathbf{R}_{+}\right)$représentable dans la forme (2), où les $\vartheta_{j}$ sont des fonctions concaves satisfaisant (3) pour $j=1$, 2. Pour $0<\rho<\infty$, nous supposons, de plus, que $\rho(r) \in C^{3}\left(\mathbf{R}_{+}\right)$et (4) est vrai, et pour $\rho=0$, nous supposons aussi que $\vartheta_{2} \equiv 0$ et (5)-(6) sont vrais. Nous notons la classe de tous ces o.p.'s par $\mathcal{A}_{\rho}, 0 \leqslant \rho<\infty$. Nous notons $\mathcal{A}_{0}^{*}$ la sous classe de $\mathcal{A}_{0}$ des fonctions $\rho(r)$ satisfaisant (8).

D'après les résultats de Earl et Hayman [2] il est naturel de définir un o.p. infini comme une fonction $\rho(r) \in C^{3}\left(\mathbf{R}_{+}\right)$ représentable dans la forme (9) où $\vartheta$ est une fonction positive convexe telle que (10) est satisfait. Nous notons $\mathcal{A}_{\infty}$ l'ensemble des o.p.'s infinis.

Les résultats de Valiron, Levin (voir [3], ch. 1), Earl et Hayman [2] montrent que la réunion de toutes les classes $\mathcal{A}_{\rho}, 0 \leqslant \rho \leqslant \infty$, est une echelle de croissance des fonctions entières dans le sens suivant : pour toute fonction entière $f$ d'ordre $\rho, 0 \leqslant \rho \leqslant \infty$, il existe un o.p. $\rho(r)$ telle que (12) est vrai, où $V(r)=r^{\rho(r)}$. Si $f$ satisfait (12), nous allons ecrire $f \in[\rho(r), \sigma]$.

Définition 0.1. Un o.p. $\rho(r)$ est dit admissible si (11) est vrai. Un o.p. $\rho(r)$ est dit fortement admissible si, de plus, $\rho(r) \notin \mathcal{A}_{0} \backslash \mathcal{A}_{0}^{*}$.

Définition 0.2. Une fonction entière $f \in[\rho(r), \sigma]$ est dite une fonction de croissance régulière $(V, \theta)$ si (13) est vrai, où $\theta$ est une fonction non-décroissante sur $\mathbf{R}_{+}$satisfaisant à (14).

Des résultats de Clunie et Kövari [1] suit l'existence des fonctions de croissance régulière $(V, \theta)$, pour tout $\rho(r)$ fortement admissible et tout $\theta$ satisfaisant aux conditions ci-dessus (dans le cas $\rho(r) \in \mathcal{A}_{0} \backslash \mathcal{A}_{0}^{*}$, il faut la condition supplémentaire $\log r=\mathrm{O}(\theta(r))$. Les functions entières des croissance très lente ne peuvent pas être des croissance très régulière dans le sens qu'il y a des restrictions inférieure pour la fonction $\theta$ dans (13) :

Théorème 0.3. Soient $\rho(r)$ un o.p. admissible et $f$ une fonction enti'ere satisfaisant aux conditions (15). Si $f$ est de croissance règuliére $(V, \theta)$ alors, l'inegalite (16) est valable.

Notre résultat principal est le suivant :

Théorème 0.4. Soit $\rho(r)$ un o.p. admissible. Si f est de croissance régulière $(V, \theta)$, alors l'inegalité (17) est valable pour tout $|w|$ suffisamment grand.

Corollaire 0.5. Si les hypothèses du Théorème 0.3 sont satisfaites et de plus $\rho(r)$ est un o.p. fortement admissible, alors (18) est vraie.

Le théorème suivant est relié à la mesure de précision du Corollaire 0.5 .

Théorème 0.6. Soient $\rho(r)$ un o.p. fortement admissible et $V(r)=r^{\rho(r)}$. Pour un $\alpha$ avec $\frac{1}{3} \leqslant \alpha<1$, soit $\theta(r)$ la fonction définie par (19). Alors il existe une fonction entière $f$ de croissance régulière $(V, \theta)$ telle que (20) est valable.

\section{Introduction}

A maximum modulus point of an entire function $f$ is a point $w \in \mathbf{C}$ such that

$$
|f(w)|=M(|w|, f), \quad \text { where } M(r, f)=\max _{|z|=r}|f(z)|, 0 \leqslant r<\infty .
$$

Denote by $R(w, f)$ the distance between a maximum modulus point $w$ and the zero set of $f$.

Lower estimates of $R(w, f)$ play an important role in Macintyre's version [4] of the Wiman-Valiron theory and its further generalizations. These estimates are expressed in terms of $M(r, f)$ and valid outside of some exceptional set 
of values of $|w|$. In [5] we obtained lower estimates of $R(w, f)$ expressed in terms of proximate order of $f$ and valid for all sufficiently large values of $|w|$.

In almost all applications of the entire functions theory (see, e.g., [3]), one deals with functions of regular growth. Therefore it is natural to study $R(w, f)$ in this case. For functions $f$ of finite positive order, we proved in [5] that the decay of $R(w, f)$ as $w \rightarrow \infty$ is slower than in general case, and the slower the more regular the growth is.

In this Note we obtain more general results applicable also to functions $f$ of either infinite or zero order and show that they can be considered as sharp in some sense.

Our results are stated in terms of proximate orders. According to Levin's definition ([3], p. 39-41), a strong p.o. is a function $\rho(r) \in C^{2}\left(\mathbf{R}_{+}\right)$representable in the form

$$
\rho(r)=\rho+\left(\vartheta_{1}(\log r)-\vartheta_{2}(\log r)\right)(\log r)^{-1}, \quad r \geqslant r_{0}>1,
$$

where $0<\rho<\infty$, and $\vartheta_{j}$ are concave functions of $C^{2}\left(\mathbf{R}_{+}\right)$satisfying conditions $(j=1,2)$ :

$$
\lim _{x \rightarrow \infty} \vartheta_{j}(x)=\infty ; \quad \lim _{x \rightarrow \infty} \vartheta_{j}(x) / x=0 ; \quad \lim _{x \rightarrow \infty} \vartheta_{j}^{\prime \prime}(x) / \vartheta_{j}^{\prime}(x)=0 .
$$

For $0<\rho<\infty$, we denote by $\mathcal{A}_{\rho}$ the class of all strong p.o.'s $\rho(r) \in C^{3}\left(\mathbf{R}_{+}\right)$satisfying the additional condition

$$
\lim _{r \rightarrow \infty} \rho^{(k)}(r) r^{k} \log r=0, \quad k=1,2,3 .
$$

For $\rho=0$, we introduce the class $\mathcal{A}_{0}$ of all strong p.o.'s representable by (2) with $\rho=0, \vartheta_{2} \equiv 0$ and $\vartheta_{1}$ satisfying, besides (3), conditions:

$$
\begin{aligned}
& \vartheta_{1}^{\prime \prime}(x)+\left(\vartheta_{1}^{\prime}(x)\right)^{2}>0, \quad x \geqslant x_{0}>0 ; \\
& \vartheta_{1}^{\prime \prime \prime}(x)\left(\vartheta_{1}^{\prime}(x)\right)^{-3}=\mathrm{O}(1), x \rightarrow \infty ; \quad \lim _{x \rightarrow \infty} x^{-1} \exp \vartheta_{1}(x)=\infty .
\end{aligned}
$$

For studying entire functions $f$ of zero order such that

$$
\limsup _{r \rightarrow \infty}(\log r)^{-2} \log M(r, f)=\infty,
$$

we will also use the subclass $\mathcal{A}_{0}^{*}$ of $\mathcal{A}_{0}$ consisting of $\rho(r)$ satisfying additional conditions:

$$
2 \vartheta_{1}^{\prime \prime}(x)+\left(\vartheta_{1}^{\prime}(x)\right)^{2}>0, \quad x \geqslant x_{0}>0 ; \quad \limsup _{x \rightarrow \infty} x^{-2} \exp \vartheta_{1}(x)=\infty .
$$

For the case of infinite order the following definition of p.o. naturally follows from results of Earl and Hayman [2]. A function $\rho(r) \in C^{3}\left(\mathbf{R}_{+}\right)$is called an infinite p.o. if it is representable in the form

$$
\rho(r)=\vartheta(\log r) / \log r, \quad r \geqslant r_{0}>1,
$$

where $\vartheta$ is a positive convex function satisfying conditions:

$$
\lim _{x \rightarrow \infty} \vartheta^{\prime}(x)=\infty ; \quad \lim _{x \rightarrow \infty} \vartheta^{(j)}(x)\left[\vartheta^{\prime}(x)\right]^{-j}=0, \quad j=2,3 .
$$

We denote by $\mathcal{A}_{\infty}$ the class of all infinite p.o.'s.

Definition 1.1. We say that a p.o. $\rho(r)$ is admissible if

$$
\rho(r) \in \bigcup_{0 \leqslant \rho \leqslant \infty} \mathcal{A}_{\rho}
$$

We say that a p.o. $\rho(r)$ is strongly admissible if it is admissible and, moreover, $\rho(r) \notin \mathcal{A}_{0} \backslash \mathcal{A}_{0}^{*}$.

Note that the set of all admissible p.o.'s is a scale of growth of entire functions in the following sense: for arbitrary entire function $f$ there exists an admissible p.o. $\rho(r)$ such that

$$
0<\sigma:=\lim _{r \rightarrow \infty}(V(r))^{-1} \log M(r, f)<\infty,
$$

where $V(r):=r^{\rho(r)}$. If an entire function $f$ satisfies (12), we will write $f \in[\rho(r), \sigma]$. 
Definition 1.2. We say that an entire function $f \in[\rho(r), \sigma]$ is a function of $(V, \theta)$-regular growth if

$$
\log M(r, f)=\sigma V(r)+\mathrm{O}(\theta(r)),
$$

where $V(r)=r^{\rho(r)}$ and $\theta$ is a positive non-decreasing function on $\mathbf{R}_{+}$satisfying conditions:

$$
\theta(r)=\mathrm{o}(V(r)), \quad \theta\left[r \exp \left(\frac{V(r)}{r V^{\prime}(r)}\right)\right]=\mathrm{O}(\theta(r)), \quad r \rightarrow \infty .
$$

Existence of entire functions of $(V, \theta)$-regular growth, for each strongly admissible p.o. $\rho(r)$ and each $\theta$ satisfying (14) (in the case $\rho(r) \in \mathcal{A}_{0} \backslash \mathcal{A}_{0}^{*}$ under additional assumption $\log r=\mathrm{O}(\theta(r))$ ), follows from results of Clunie and Kövari (see [1], Theorems 2 and 4). Note that entire functions of very slow growth cannot have 'very regular growth'. We proved the following fact:

Theorem 1.3. Let $\rho(r)$ be an admissible p.o. and let $f$ be an entire function satisfying condition

$$
\log M(r, f)=\mathrm{o}\left(\log ^{2} r\right), \quad r \rightarrow \infty .
$$

If $f$ is of $(V, \theta)$-regular growth, then

$$
\limsup _{r \rightarrow \infty} r V^{\prime}(r) \sqrt{\theta(r) / V(r)}>0 .
$$

\section{Main results}

Theorem 2.1. Let $\rho(r)$ be an admissible p.o. and let $V(r)=r^{\rho(r)}$. If $f$ is of $(V, \theta)$-regular growth, then, for all sufficiently large $|w|$, the inequality holds

$$
\frac{R(w, f)}{|w|} \geqslant 1-\exp \left\{-\frac{C}{|w| V^{\prime}(|w|)} \sqrt{\frac{V(|w|)}{\theta(|w|)}}\right\},
$$

where $C$ is a positive constant.

Corollary 2.2. If conditions of Theorem 2.1 are satisfied and, moreover, $\rho(r)$ is a strongly admissible p.o., then

$$
\liminf _{|w| \rightarrow \infty} R(w, f) V^{\prime}(|w|) \sqrt{\theta(|w|) / V(|w|)}>0 .
$$

The following result relates to sharpness of Corollary 2.2.

Theorem 2.3. Let $\rho(r)$ be a strongly admissible p.o. and let $V(r)=r^{\rho(r)}$. Given $\frac{1}{3} \leqslant \alpha<1$, define

$$
\theta(r)=V(r)\left(r V^{\prime}(r)\right)^{\alpha-1} .
$$

There exist an entire function $f$ of $(V, \theta)$-regular growth such that

$$
\liminf _{|w| \rightarrow \infty} R(w, f) V^{\prime}(|w|) \sqrt{\theta(|w|) / V(|w|)} \leqslant \pi .
$$

\section{References}

[1] J. Clunie, T. Kövari, On integral functions having prescribed asymptotic growth. II, Canad. J. Math. 20 (1968) 7-20.

[2] J.P. Earl, W.K. Hayman, Smooth majorants for functions of arbitrarily rapid growth, Math. Proc. Cambridge Philos. Soc. 109 (1991) 565-569.

[3] B.Ya. Levin, Distribution of Zeros of Entire Functions, Amer. Math. Soc., Providence, RI, 1980.

[4] A.J. Macintyre, Wiman's method and "flat regions" of integral functions, Quart. J. Math. 9 (1938) 81-88.

[5] I.V. Ostrovskii, A.E. Üreyen, Distance between a maximum modulus point and zero set of an entire function, Complex Variables 48 (2003) 583-598. 